\title{
Research on the Construction of Subway Information Service Platform Based on Mobile Internet
}

\author{
Jinhuan Wang, Yan Li, Qing Lin, Chun Jiao \\ School of Intelligence Science and Information Engineering \\ Xi'an Peihua University, China \\ 360635476@qq.com
}

Keywords: Mobile internet; New structure; Service approach

\begin{abstract}
Based on the in-depth study of the traditional subway information service management system, the paper analyzes and researches the new requirements of users for subway information services, and combines the latest research results of mobile internet technology to build a new architecture of a smart subway information service platform based on mobile internet technology. The subway information service system has become more diversified, more efficient, better quality, and more intelligent.
\end{abstract}

\section{Introduction}

With the rapid growth of the big data industry, the combination of emerging IT technologies such as big data and mobile Internet and new business models is changing people's life. The introduction of mobile Internet technology not only can deepen the informatization construction of urban transportation, but also can carry out comprehensive processing and massive data analysis on the basis of automatic acquisition of traffic information, realize the intelligentization of operations in the transportation field, generate optimization solutions to requirements at the fastest rate, and provide residents with safer, more convenient, comfortable, efficient and smart travel services.

\section{Problems with Subway Information Services}

With the application of computer and Internet technologies, Xi'an Metro Information Service mainly has the following problems:

(1) The range and route of passenger service information, especially emergency information release, are limited, and it is not convenient for propaganda of passenger service information;

(2) The traditional manual service form has been difficult to adapt to, and the station needs to install the "Intelligent Screen" (mobile intelligent terminal) that has the function of interacting with the passengers and facilitating passengers to self-check various service information;

(3) At present, no system for intelligent information service management has been established, lack of technical support, lack of induction and analysis of subway information management, and inadequate provision of information for decision-making;

(4) Data heterogeneity. The vast majority of passenger-related information is recorded in the metro information system. The huge potential value knowledge is contained in these large and heterogeneous metro database information systems, lacking unified and standardized management and utilization.

\section{The Main Contents of the Research on the Information Service Platform}

Based on the in-depth study of the traditional subway information service system, the research and analysis were conducted. Based on the existing problems, the mobile internet technology was introduced to apply the latest research results of the big data center and data mining to the smart information service of subways. Passengers are provided with "anytime, anywhere" subway service, and passengers are allowed to experience the convenience and speed of "Smart Subway". The main content of this paper's research includes the following five aspects. 


\subsection{Analyze and Research the Application Requirements of Metro Information Service}

With the development of society and the rapid growth of the urban economy, people's demand for convenient and quick travel continues to increase. The choice of a quick and efficient travel plan is increasingly valued by residents and tourists. Coupled with the rapid development of the mobile Internet, the use of intelligent terminal devices to obtain real-time information on public transportation has become a hot demand. Services include travel pamphlet, local services, online media entertainment, and subway Internet finance.

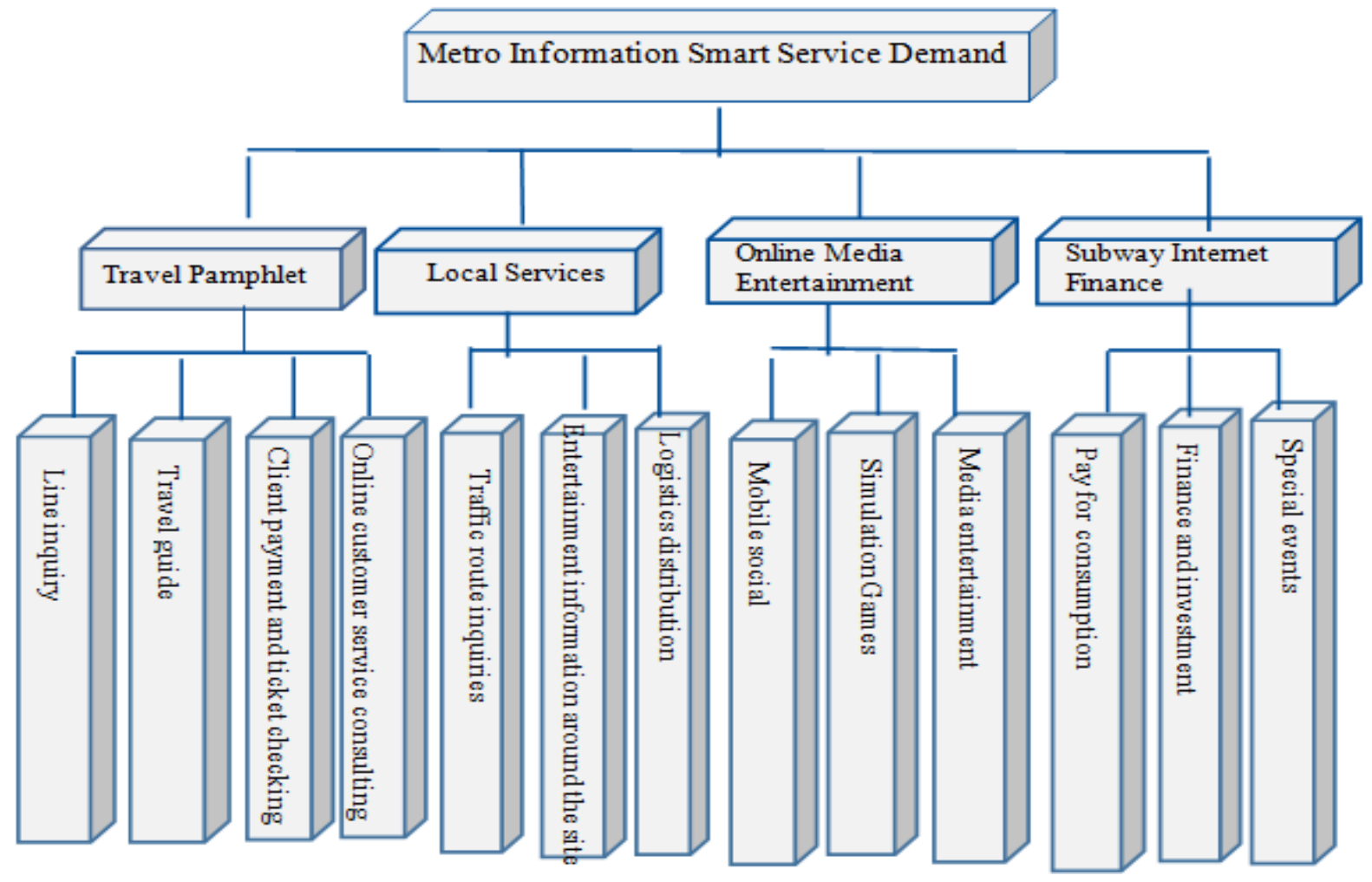

Figure 1. Metro information smart service demand organization chart

\subsection{Study the Application Thinking Mode of Smart Subway}

The continuous emergence and penetration of modern information technologies such as big data, Internet of Things, cloud computing, and mobile Internet have accelerated the intelligent development of transportation. The following four thinkings point the way for the study of subway smart information services.

(1) User Thinking: That is to say, consider the 'user-centered' design in all aspects of the new service system construction, which is the core of Internet thinking.

(2) Big Data Thinking: In the era of big data, the construction of a service system involves big data thinking. Fully considering the diversified and personalized information and service needs of travelers, it classifies and analyzes the large amount of information by means of big data center technology, correlation analysis technology.

(3) Relevance Analysis and Data Mining Thinking: The Internet's thinking is an open, shared, and win-win thinking, which means that the company must be built as an open, multi-winning, mutually beneficial ecosystem.

(4) Distributed Computing Thinking: For the data mining needs of the metro information service system, the mining algorithm needs to have good scalability and parallelism. The Hadoop big data analysis technology and Spark memory computing technology can be cited to provide the underlying support for data mining.

\subsection{Building a New Architecture of Services Based on Big Data}

Based on the in-depth study of the existing subway service architecture, combined with mobile 
Internet technology, the latest research results of big data centers and data mining are introduced into the subway service model system, making the subway service system more diversified, more efficient,better quality and more intelligent. The new architecture is shown in Fig. 2 .

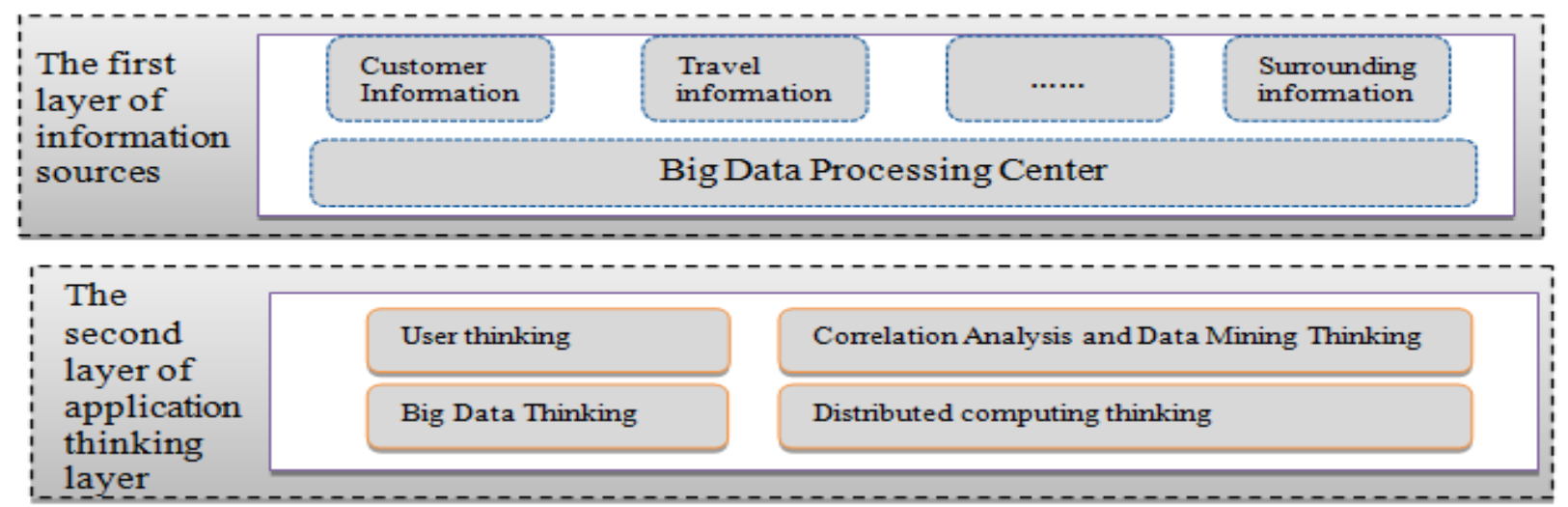

Mobile Internet technology

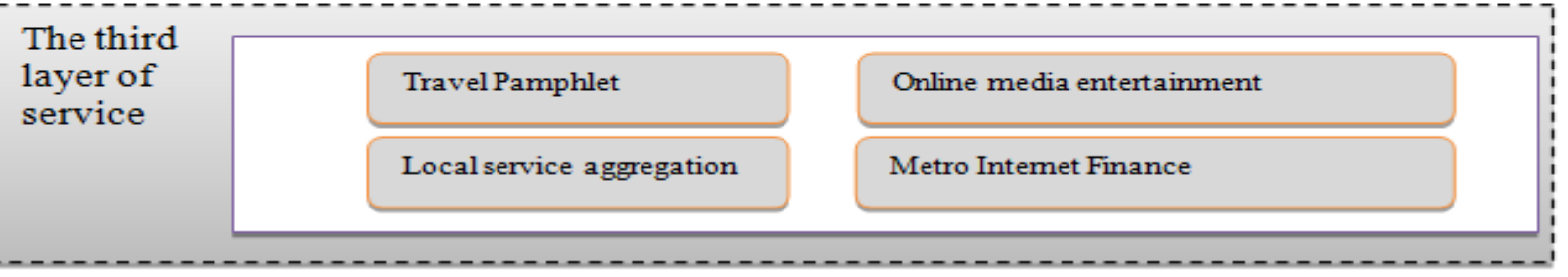

Figure.2. New architecture of subway information service platform based on Mobile internet

The main content of this architecture study includes the following three aspects:

(1) Sources of information: Including WeChat ticketing, real-time passenger flow, subway news, video information, fare inquiries, route maps, travel information, the first and last metro, around the station, lost and found items, auto passenger transport, high-speed rail inquiries, etc, which are big data information processing center.

(2) The Application Thinking Layer: includes big data center technology, correlation analysis technology and data mining.

(3) The Functional Areas of Information Services: include information modules such as travel pamphlet, subway internet finance, local service gatherings, and online media entertainment.

\subsection{The Establishment of Smart Subway Information Service Cloud Platform Model}

The basic information service security platform includes: SaaS platform, PaaS platform application server, operating system, virtual resource pool, and physical resources.

The basic information service security platform includes: SaaS platform (data query, data mining, etc.), PaaS platform (programming interface, data management, template editing, etc.), application server, operating system, virtual resource pool, and physical resources.

\subsection{Xi'an Smart Subway Information Service Platform Terminal Research}

Xi'an Subway Information Service will realize intelligent services. The contents of terminal research mainly include: Research on Information Enquiry of Multimedia Smart Self-service Machine (referred to as Great Wisdom). Passengers can search for subway bus travel plans and city service information by touching, and access specific information on various public service facilities around the subway station;" Big Wisdom" can directly extract data from the cloud and can calculate passengers' traffic transfer plans in a short period of time, such as the best transportation routes and the shortest transit time for different modes of transportation such as subways, buses, and walking; "Great Wisdom" humanity Functional design research, such as subway + government affairs, hospitals, schools, food, hotels, etc. 


\section{Technology Used in the Construction of Subway Information Service Platform}

Data mining related technologies are used to study data clustering analysis, algorithms and modeling. For example, Bayes model, artificial neural network, random forest algorithm, decision tree theory, D-S evidence theory, clinical decision index matrix theory, etc. It may involve multiple models and algorithms in one type of application; study fast retrieval and processing of big data (infrastructure construction, large-capacity metro data organization, storage and indexing technology, high-concurrency access to data and rapid extraction, etc.); and study the software design of the information security basic platform, to ensure that metro data is not stolen and modified by the outside world during its use, and to establish corresponding data encryption technologies and data access authorization mechanisms.

\section{Conclusions}

The goal of this paper is to use the latest mobile Internet technology and combine big data analysis to research Xi'an Metro Information Services, based on data mining technology, to reconstruct business information of big data and establish a unified business view. From multiple perspectives on the subway, the data of the smart information service system is further explored, and the rules hidden behind the data are excavated, which can effectively assist passengers in making quick decisions on information, effectively forecast information services, provide data support for decision-making of the leadership to obtain the new way of information service of Xi'an wisdom subway and provide people with better services. The research of Xi'an's smart subway information service based on big data analysis is of great value.

\section{Acknowledgment}

This work is supported by 2017 Xi'an Social Science Planning Fund Xi'an Peihua Special Project(No.17PH07) and Special scientific research project of the Shaanxi Provincial Education Department in 2015, support project (No.15JK2091), all support is gratefully acknowledged.

\section{References}

[1] Huang Lanyan. The Application Conception of Big Data in Urban Planning.Architectural \& Planning Design,2017(23):16-17.

[2] Yang Shanlin, Zhou Kaile. Management Issues in Big Data-A View of Resources Based on Big Data.Journal of Management Science,2015(5).1-8.

[3] Li Wenlian,Xia Jianming. Business Model Innovation Based on "Big Data".China's Industrial Economy,2013(5).83-95.

[4] Fan Ling. Study on optimization of MOOCs large data clustering method in wireless network. Computer simulation., 2016(7).435-440.

[5] Li Zhengjie. Research on SVM_KNN Classification Algorithm Based on Hadoop Platform.Computer Technology and Development, 2016(3).75-80.

[6] Su Yijuan. Fast KNN Classification Algorithm under Big Data.Computer Applications Research, 2016(4).1003-1007. 